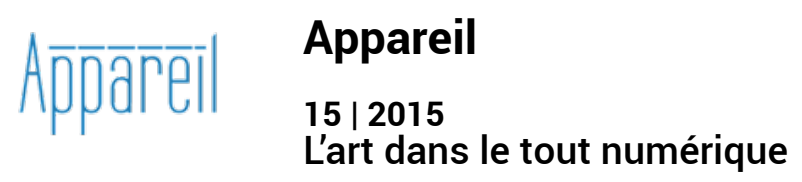

\title{
Dans la douleur des autres
}

Désastre, mobilité et culture numérique

\section{Richard Bégin}

\section{(2) OpenEdition}

\section{Journals}

Édition électronique

URL : http://journals.openedition.org/appareil/1259

DOI : 10.4000/appareil. 1259

ISSN : 2101-0714

Éditeur

MSH Paris Nord

Référence électronique

Richard Bégin, "Dans la douleur des autres », Appareil [En ligne], 15 | 2015, mis en ligne le 16 juillet 2015, consulté le 09 août 2020. URL : http://journals.openedition.org/appareil/1259 ; DOI : https:// doi.org/10.4000/appareil.1259

Ce document a été généré automatiquement le 9 août 2020.

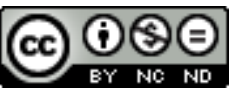

Appareil est mis à disposition selon les termes de la Licence Creative Commons Attribution - Pas d'Utilisation Commerciale - Pas de Modification 4.0 International. 


\title{
Dans la douleur des autres
}

\author{
Désastre, mobilité et culture numérique
}

\author{
Richard Bégin
}

1 Je présenterai ici l'un des trois axes de recherche d'un programme beaucoup plus vaste qui a pour titre « Désastre et culture numérique : les productions actuelles de l'espace traumatique ». Comme son titre le suggère, ce programme a pour objectif général d'étudier en quoi les technologies numériques favorisent l'émergence d'une figuration inédite du désastre. Plus précisément, il s'agit de voir en quoi les différents appareils médiatiques issus de la technologie numérique nous permettent de nous figurer autrement le désastre, la violence et le cataclysme ; autrement que ce à quoi nous ont habitués, notamment les appareils issus de la technologie analogique, de la photographie au cinéma. Il s'agit en outre de comprendre comment cette figuration du désastre parvient à engendrer un nouvel espace traumatique qui se distingue de celui que révèle la distanciation représentative spécifique aux témoignages écrits, graphiques ou photographiques de la guerre, de la catastrophe et autres calamités. Les appareils médiatiques actuels - du téléphone "intelligent», à l'Internet, au cinéma 3D, en passant par le jeu vidéo - inaugurent pour leur part une production d'espaces qui leur est propre, susceptible en cela de redéfinir la figure du désastre. Une figure redéfinie, entre autres, en raison d'une culture numérique qui favorise davantage les processus d' immersion que la logique habituelle de l'émersion, soit de ce qu'implique l'argument courant concernant ce qui émerge, se dégage ou se manifeste d'une représentation. Aussi, si la culture analogique ainsi que ses clichés insoutenables desquels se dégagerait un savoir de la guerre et de ses souffrances nous apprennent, comme le laisse entendre Susan Sontag ${ }^{1}$, à nous désoler devant la douleur des autres, la culture numérique nous apprendrait quant à elle à vibrer dans la douleur des autres.

2 Ce programme de recherche a pour objectif plus spécifique de développer l'hypothèse que la production d'espace au sein de la culture numérique actuelle se caractérise par trois paradigmes de spatialisation. Lesquels représentent chacun les trois axes de recherche du programme. Dans le cadre de cette recherche je développerai ces trois axes de manière à souligner trois différentes dynamiques d'appareils qui chacune, à sa manière, dévoile un mode d'apparition de la spatialité, soit, plus précisément, un 
dévoilement suivant une dynamique de la mobilité (pour ce qui concerne l'Internet et le téléphone "intelligent»), de la synthèse (pour ce qui concerne l'animation et le cinéma 3D) et du ludisme (pour ce qui concerne le CD-Rom et le jeu vidéo). Ces trois modes d'apparition de la spatialité relèvent, dans l'ordre, d'un paradigme de la circulation, de la virtualisation et de la remédiation. En somme, qu'il s'agisse de la mise en circulation de scènes de dévastation (via la mobilité du dispositif portable), d'une virtualisation du paysage cataclysmique (via l'imagerie de synthèse - ou le CGI [computer-generated imagery] - au cinéma) ou d'une remédiation vidéo-ludique de l'expérience catastrophiste (via l'intermédiaire récréatif et l'interface du jeu vidéo), les appareils médiatiques actuels contribuent manifestement à composer un nouvel imaginaire du désastre. C'est ce nouvel imaginaire lié à la production d'espaces dit « virtuels » qui m'apparaît apte à composer une iconosphère du désastre susceptible, non plus de définir les lieux d'émergence du trauma, mais, si j'ose dire, de circonscrire les lieux d'immergence dans l'espace traumatique.

\section{Vers une « mobilité » du désastre}

3 Je limiterai ici mon analyse au premier axe de recherche, soit à l'espace traumatique tel qu'il est produit par l'appareil médiatique mobile de $3^{\mathrm{e}}$ ou de $4^{\mathrm{e}}$ génération, plus communément appelé «téléphone intelligent». Cet appareil médiatique muni d'une caméra prolonge dans le régime de la visualité une expérience de l'espace "mobile », ou de la mobilisation spatiale, à laquelle nous avait déjà habitué le téléphone cellulaire «traditionnel ». Malgré l'absence de caméra intégrée, ce dernier appareil n'en a pas moins jeté les bases d'une nouvelle production d'espace, suivant laquelle il n'y avait désormais d'emplacements que comme fluctuations des places. Pour nous faire une idée de ce que cette production en devenir impliquait déjà en terme d'immergence dans l'espace traumatique, songeons seulement à cette conversation ayant eu lieu, un certain 11 septembre 2001, entre un homme et sa femme, passagère du vol United 93, se sachant condamnée à mourir dans les minutes à venir. En analysant froidement ce moment dramatique - et, dans une certaine mesure, inaugural -, il nous apparaît clair que celui-ci participe d'une figuration du désastre caractéristique d'une culture numérique privilégiant l'abolition graduelle de la distanciation représentative. Le trauma lié aux attentats prend dans ce cas-ci les formes atmosphérique et aérienne d'une circulation - sonore - de l'expérience catastrophiste. Par la même occasion, c'est le désastre même du 11 septembre 2001 qui acquiert de la sorte une forme de «mobilité » relationnelle qui, on le devine, aurait atteint un tout autre degré de traumatisme si la dame en question avait eu en sa possession un "téléphone intelligent $»$.

On a alors peine à dissimuler notre "déception » de ne pouvoir posséder des images provenant de l'intérieur du fuselage, bref, de vibrer dans la souffrance et l'angoisse des autres. Cette déception, s'il en est une, est symptomatique d'une culture de l'image qui a progressivement glissé d'une appréhension intuitive de l'espace à son incessante production matérielle. Dans leur introduction au livre Mediaspace. Place, Scale and Culture in a Media Age, les auteurs Nick Couldry et Anna McCarthy placent en exergue deux citations, la première de Rainer Maria Rilke, la seconde de Michel Foucault :

Par tous les êtres se déploie l'espace unique, espace intime au monde... ${ }^{2}$

[...] nous vivons à l'intérieur d'un ensemble de relations qui définissent des

emplacements irréductibles les uns aux autres et absolument non superposables. ${ }^{3}$ 
Ces deux citations illustrent selon Couldry et McCarthy le passage d'une culture à une autre, soit, le passage d'une culture visuelle qui appréhendait l'espace comme le résultat d'une image intérieure ou d'une métaphysique de la distanciation, à une culture visuelle qui a vu naître du concept de distance son déploiement matériel ainsi que son explicitation médiatique. Un déploiement ayant redistribué à l'horizontal des pouvoirs dont on n'avait jusqu'alors qu'une intuition verticale. Une sécularisation spatiale, bref, qu'évoque, par exemple, l'espace panoptique chez Foucault qui explicite à la fois le partage et la médiation des pouvoirs. Mais ces deux citations évoquent de manière plus dramatique encore le passage d'une catégorie conceptuelle de l'espace héritée de Kant - l'espace comme possibilité de coexistence - aux conditions «modernes» de matérialisation relationnelle de celui-ci - de la coexistence comme possibilité d'espace. Cette matérialisation relationnelle se concrétisera dès la seconde moitié du $\mathrm{XIX}^{\mathrm{e}}$ siècle grâce à l'appareil médiatique de $1^{\text {er }}$ type (l'appareil de reproduction photographique et cinématographique) en ce qu'il est susceptible de générer un espace analogique partagé, justement, entre deux «emplacements irréductibles », celui de la réalisation et celui de la réception. Dans leur lecture de Rilke et de Foucault, Couldry et McCarthy prolongent en cela l'idée qui sous-tend l'ensemble des travaux d'Henri Lefebvre sur l'espace "moderne ", soit que tout espace est avant tout lié à sa production; et que de cette production se dégage une culture spécifique. Dans ce cas-ci, une culture analogique.

6 Une autre révolution « culturelle » de l'image interviendra, cependant, dès l'instant où l'appareil médiatique ne se contentera plus de perpétuer une "reproductibilité technique » de l'espace, pour reprendre les termes de Walter Benjamin, mais intègrera de surcroît les rouages d'un «système de diffusion ». Le broadcast, comme on l'appelle couramment, aura introduit une nouvelle manière de se figurer l'espace, autrement que par sa seule «analogie» ou «reproductibilité technique». Les chaînes d'information continue, par exemple, auront favorisé pour leur part la production d'un espace entrainnant ce que Paddy Scannell nomme le «redoublement de l'emplacement » :

Public Events now occur, simultaneously, in two different places: the place of the event itself and that in which it is watched and heard. Broadcasting mediates between the two sites. $^{4}$

7 Ce «redoublement de l'emplacement " provoqué par un tel «système de diffusion » aura eu pour résultat de produire un espace simultanément "partagé », via l'intermédiaire télé, entre le lieu de sa réalisation et celui de sa réception. Se dégagera dans ce cas-ci une culture de nature télévisuelle. Mais à ce redoublement simultané de l'emplacement que permet l'appareil médiatique de $2^{\text {e }}$ type - de la radio à la télévision - s'ajoute encore une autre révolution «culturelle» de l'image; une rencontre du $3^{\text {e }}$ type, en somme, introduite par la numérisation de l'information, et dont le téléphone portable « intelligent » s'avère être l'occurrence privilégiée.

8 Ce sera bien évidemment grâce à l'introduction du transfert numérique d'informations que l'appareil médiatique mobile permettra désormais à tout un chacun de partager simultanément une expérience quelle qu'elle soit. De même, l'appareil numérique mobile génère-t-il dès lors une pluralité «fluide » des places qui nous oblige, de fait, à reconsidérer ce qu'implique dorénavant la « couverture » d'un événement, ainsi que sa communication. Plus exactement, en raison de la mobilité même de l'appareil, l'événement s'avère être moins « couvert », que pratiqué, littéralement. L'événement ne 
se contente plus ici d'être transmis par l'intermédiaire télé ou via le "système de diffusion " - le broadcasting -, il se construit au gré de ce qu'Henri Lefebvre, dans La production de l'espace, appelait une pratique spatiale ${ }^{5}$. Autrement dit, le désastre comme tel, par exemple, se constitue désormais dans la mobilité, suivant une production d'espace intimement liée à l'appropriation somatique de l'événement, soit, si l'on veut, à sa prise «à-bras-le-corps ». C'est cette appropriation somatique qui commande toute notre attention en ce qu'elle introduit une culture visuelle de la catastrophe qui participe de près à ce que Daniel Bougnoux appelle une "crise de la représentation ", mais que nous étudierons plutôt ici comme une " prise de la présentation ».

\section{La pratique spatiale du médiateur}

Ce n'est qu'à partir du 26 décembre 2004, date du violent tremblement de terre ayant secoué le littoral indonésien, qu'on aura pu prendre la mesure exacte de ce qu'implique pour notre "révolution" culturelle de l'image cette pratique spatiale inédite. Au moment où le tsunami frappait la province de Phuket, nombreux étaient les touristes sur place en mesure d'immortaliser via leur téléphone cellulaire les images d'un désastre en train de se produire. Bien avant qu'elles n'alimentent les chaînes d'information télévisée habituelles, ces images pouvaient être visionnées par tout un chacun, peu importe le lieu, sur plusieurs sites web et via de nombreux réseaux sociaux. Aussi, l'espace du désastre pouvait-il être partagé sans qu'il ne fût au préalable composé et produit par ces "instaurateurs de discursivité» (M. Foucault) que représentent les chaînes d'information continue. Par conséquent, c'est toute une crise humanitaire spatiale qui pouvait de la sorte être «mobilisée » et transmise d'individu à individu bien avant que celle-ci ne fût traduite par l'intermédiaire télé en un état de fait géopolitique. La libre circulation sur Internet de ces images dans les minutes qui ont suivi l'événement nous rappelle ainsi à l'esprit une idée avancée par Gabriel Tarde dès la fin du XIX siècle suivant laquelle toute question sociale devrait d'abord être appréhendée comme un «fluide en circulation ». L'événement du 26 décembre 2004 a bel et bien eu une incidence sociale en ce que le raz-de-marée " a créé un nouvel état de société, un autre espace, inédit et durable, qui perdure bien après le reflux. $»^{6}$. Or, cet autre espace, comme on le constate, ne peut seulement être réduit à ce qui a pour origine physique la catastrophe naturelle; cet autre espace est également le produit d'une médiation.

Les images de la catastrophe indonésienne de décembre 2004 participent ainsi de la constitution d'un imaginaire du désastre qui ne concerne plus en dernière instance la diffusion médiatique au sein des chaînes d'information continue d'un certain "état de fait» géopolitique. L'imaginaire du désastre concerne ici, en première instance, l'appropriation somatique de l'événement ainsi que la circulation de celui-ci via l'appareil qui en permet la captation et la diffusion. Une appropriation-captation et une circulation-diffusion qui s'effectue grâce à ce que Bruno Latour, dans sa théorie de l'acteur-réseau, nomme le médiateur, soit l'entité dynamique impliquant une modification et une transformation du sens des éléments qu'elle est censée transporter ${ }^{7}$. L'appareil numérique mobile s'inscrit ici comme le médiateur privilégié à l'origine de la production d'un espace traumatique se différenciant à la fois de l'élément catastrophique - puisqu'il le médiatise - et de sa remédiation télévisuelle puisqu'il le précède nécessairement. L'appareil provoque ainsi une modification de 
l'événement en ce qu'il en permet la "pratique » relationnelle, sans toutefois l'inscrire dans le registre de la représentation symbolique. L'événement se prolonge alors en un réseau ou, pour reprendre les termes de Peter Sloterdijk, en un "déploiement multifocal, multiperspectiviste et hétérarchique » de vibration traumatique. En ce sens, l'appareil mobile s'avère être le médiateur privilégié d'un espace traumatique immergeant, lequel précède et excède à la fois la simple remédiation télévisuelle d'images desquelles se dégagerait, par exemple, un discours ayant pour sujet la création d'un nouvel état de société ou d'une nouvelle ère géopolitique.

11 Selon Bruno Latour, le médiateur se distingue de l'intermédiaire en ce que ce dernier a pour effet de plonger la dynamique de l'événement dans le savoir-faire et la transmission, et comme mentionné plus haut, la remédiation, au sens où l'entendent Jay David Bolter et Richard Grusin ${ }^{8}$. Une remédiation à laquelle correspond la diffusion télévisuelle des images de la catastrophe du moment où cette transmission, par son effet de boucle et de répétition, stabilise la dynamique chaotique - et, en cela, traumatique - de l'événement au sein d'une forme de métaphysique sociale qui ne nous fait plus voir de l'espace « désastré » qu'un « état de fait » ou la cause d'une profonde transformation géopolitique. Un "état de fait» que l'on peut traduire comme le résultat d'une représentation médiatique, voire d'un broadcasting de la réalité catastrophique. C'est ce même résultat qui permet l'établissement commun d'une époque que l'on reconnaît être celle de la globalisation.

Or, du moment où il est pris "à-bras-le-corps ", notamment grâce à la mobilité de l'appareil numérique mobile, l'espace traumatique du désastre excède le broadcasting dans la mesure où il est littéralement produit par ce que fait faire le mobile à son utilisateur. Plus exactement, cet espace traumatique devient le pur produit d'une pratique individuée - et non d'une remédiation dividuelle, commune et publique - de l'événement ; d'une pratique différentielle, bref, se conjuguant à une technologie qui en permet, de fait, la circulation, de médiateur en médiateur. Du coup, il y a bel et bien une possibilité d'espace antérieur à sa transmission "globale " ainsi qu'à sa répétition publique, et cette possibilité se manifeste non seulement par la mise en réseau de l'événement, mais par l'articulation d'une agglomération ou d'une "association" technologique en perpétuel devenir. C'est cette même "association » que l'on peut traduire, avant qu'elle ne fasse "société », comme le résultat d'une explicitation de la réalité catastrophique, soit comme la formulation visuelle et co-existentielle d'un «climat» de terreur. Un résultat qui illustre pour sa part les contours d'une époque que l'on reconnaît être celle, cette fois, de la blogalisation.

13 Un grand nombre d'images circulant sur Internet nous permettraient, une fois colligées, de mieux saisir encore les contours de cette explicitation de la réalité catastrophique, entendue ici comme la formation visuelle et associative d'un "climat " de terreur. Qu'il s'agisse des images tournées par un militaire américain au moment du séisme en Haïti le 12 janvier 2010 ou de celles tournées chez lui par un jeune japonais le 11 mars 2011, ces images nous invitent toutes à nous immerger dans l'espace traumatique de la catastrophe. Mais ces dernières introduisent également un régime d'imagéité spécifique duquel s'exprime une empathie envers l'angoisse et la terreur des autres. Ce régime d'imagéité évoque de la sorte une esthétique particulière qui excède le simple "redoublement de l'emplacement» que rend possible la représentation médiatique de la réalité; il énonce plutôt dans la «mobilité» les contours pragmatiques d'une réalité polymorphe en permanente délocalisation. La pluralité des 
places ainsi permise par l'appareil numérique mobile a alors pour conséquence ultime de composer une sphère iconique - une iconosphère - inédite. Celle-ci aurait pu, il y a fort longtemps, être définie par Pascal en ces mots : «C'est une sphère infinie, dont le centre est partout, la circonférence nulle part. » (Blaise Pascal, Pensées, 1670). C'est que les images que rend possible l'appareil numérique mobile ont d'abord et avant tout pour but de circuler et de s'articuler d'acteurs en acteurs, de médiateurs en médiateurs. Certes, ces images sont régulièrement prises dans les filets de l'intermédiaire - une prise qui mérite également toute notre attention en ce qu'elle engage ces images dans l'ordre symbolique du discours - ; néanmoins ces images « somatiques » n'en instaurent pas moins, selon moi, une nouvelle esthétique du désastre. Une esthétique qui commande une véritable théorie de l'articulation technologique de l'image délocalisée, bref, de ce que j'appellerais, suivant les termes issus de la théorie «sphérologique » de Peter Sloterdijk, une théorie visuelle de l'écumisation médiatique.

\section{Ouverture}

\section{Une culture visuelle de l'écumisation médiatique}

Dans le premier tome de sa trilogie des sphères, Peter Sloterdijk parle de l'écume en ces termes :

Là où tout est devenu centre, il n'existe plus de centre valide; là où tout émet, l'émetteur supposé central se perd dans le flot des messages entremêlés. Nous voyons comment et pourquoi l'ère du cercle de l'unité, l'unique, le plus grand, celui qui englobe toute chose, et celle des exégètes courbés est irrévocablement passée. L'image morphologique du monde polysphérique que nous habitons n'est plus la sphère, mais l'écume. La mise en réseau actuelle, qui encercle la terre entière - avec toutes ses excroissances dans le virtuel - ne représente donc pas tant, d'un point de vue structurel, une globalisation qu'une écumisation. ${ }^{9}$

Une théorie visuelle de l'écumisation médiatique consisterait en ce sens à rendre compte d'une esthétique de la décentralisation ou de la délocalisation imageante permise par la technologie numérique de l'appareil médiatique mobile. Bref, en ce qui nous concerne, à rendre compte d'une esthétique de l'explicitation "climatique» de la terreur et du déploiement médiatique de l'espace traumatique. Cette théorie nous permettrait plus spécifiquement d'appréhender un nouveau «mode de dévoilement » du désastre, pour parler comme Heidegger, qui aurait pour conséquence inédite d'introduire la «figuration » de celui-ci dans le registre de l'ambiant plutôt que dans le régime habituel de la représentation et de la remédiation.

Une remédiation narrative de cette « ambiance » catastrophiste sert clairement le récit de deux films populaires récents : Diary of the Dead (Georges A. Romero, 2007) et The Happening (M. Night Shyamalan, 2008). Dans ces deux cas, il nous est possible de nous figurer le désastre via l'appareil mobile permettant l'explicitation d'une réalité catastrophique de nature "épidémiologique ». Dans le film de Romero, un groupe de jeunes étudiants n'a, pour dernier contact avec le "monde extérieur», qu'une image circulant sur Internet présentant une jeune asiatique paniquée soulignant, de chez elle, la nécessité, pour se débarrasser des zombies, de viser la tête. Ce n'est pas cette information, bien qu'essentielle, qui fait la force d'une telle séquence, mais bien la manifestation de l'atmosphère médiatique qu'elle suppose. En effet, cette information s'adresse moins à la population en général, tel que le ferait une " adresse » télédiffusée en continue sur les grandes chaînes publiques, qu'elle n'institue une coexistence 
médiatique entre une multitude d'acteurs possédant tous, idéalement, un appareil numérique mobile. De sorte qu'une «ambiance» catastrophiste naît ici d'une association technologique "pré-contractuelle », comme dirait Sloterdijk, en ce qu'elle ne peut être réduite en un agir communicationnel qui dépendrait, par exemple, du langage de l'intermédiaire télévisuel. La même chose se produit dans le film de Shyamalan au moment où un petit groupe de survivants reçoit des images témoignant, de l'autre bout du pays, de la progression dramatique d'un virus mortel. Dans ces deux cas, la propagation d'un virus s'explicite - et non pas s'explique - via l'articulation "associative» des médiateurs. La réalité épidémiologique s'émancipe alors de l'intermédiaire pour n'être plus que "fluide en circulation ». De même, s'émancipant de la représentation, la figure du désastre s'énonce-t-elle dans le déploiement technologique de l'image que rend possible le téléphone «intelligent». De cette « mobilité du désastre » s'exprime alors un véritable « climat » de terreur.

On parlera dans ce cas-ci d'une culture visuelle de l'écumisation médiatique en ce que l'image du désastre qu'impliquent ces films, produit, pareil à un symptôme d'un mode contemporain de la visualité, les dimensions spatiales d'une co-isolation des médiateurs. En d'autres termes, l'espace traumatique du désastre relève dans ces deux cas de figure d'une articulation médiatique de la catastrophe qui a pour conséquence de mobiliser cette dernière dans le passage continu qui voit celle-ci circuler de médiateur isolé en médiateur isolé. C'est ce passage même qui dévoile, et explicite par la même occasion, la propagation d'un virus qui ne cessera qu'au moment où cesseront la circulation et l'articulation de sa communication. De même l'espace viral se représentet-il moins qu'il ne se présente au médiateur du moment où celui-ci ne fait qu'un avec l'espace quasi vital qu'instaure la délocalisation médiatique. Les films de Romero et de Shyamalan illustrent chacun à leur façon les torts et travers de ce nouvel espace vital, voire de ce modus vivendi numérique qui ne connaît plus de la distance face à l'événement que l'écrasement de celle-ci; un écrasement n'en permettant pas moins l'expression d'un nouveau «climat » de terreur lié, cette fois, au dévoilement d'un lieu d'immergence dans l'espace traumatique. C'est ce même lieu qui permet l'articulation de la culture numérique actuelle à ce qu'on pourrait appeler l'esthétique terroriste contemporaine.

Je noterai, pour conclure, que ce "climat » de terreur que " dévoile» la technologie de l'appareil numérique mobile trouve peut-être dans le Pocket Film son moyen d'expression le plus abouti, sinon, le plus pertinent. Le terme de Pocket Film réfère à ces films - surtout de très courts-métrages - réalisés à l'aide d'un téléphone "intelligent ", puis diffusés, pour la majorité d'entre eux, sur Internet. L'axe de recherche dont je viens ici de présenter les grandes lignes a, parmi ses objets d'étude, cette "espèce » filmique d'un nouveau genre, ou cet "usage » cinématographique inédit, qui a pour ambition première de conjuguer la poétique $\mathrm{du}$ film à celle qu'inaugurent les possibilités techniques de l'appareil numérique mobile. Un Pocket Film attirera plus particulièrement l'attention. Il s'agit de 15 août 2006 de l'artiste-web Albertine Meunier $^{10}$. Dans ce très court film de fiction, Meunier offre à la fois une remédiation narrative de l'ambiance catastrophiste liée à l'attentat terroriste, ainsi que les formes de son écumisation médiatique. Il s'agit d'un «film de poche» inspiré de la " couverture ", ou plutôt, de la "pratique " des événements survenus à Londres, le 7 juillet 2005. Dans ce film, rien ne représente un quelconque attentat ou les traces physiques d'une catastrophe quelle qu'elle soit. Seule la visualité du médium laisse s'exprimer le lieu d'immergence dans l'espace traumatique, en ce que ce lieu naît de la 
mobilité d'un désastre dont on ne peut que se figurer l'ambiance somatique, soit les formes de ce qu'il provoque comme pratique spatiale de l'effroi. C'est ce «lieu » qui commande une analyse approfondie de l'iconosphère numérique dans la mesure où cette nouvelle « sphère intermédiaire » introduit au sein de l'esthétique visuelle les formes et codes d'une culture actuelle privilégiant, comme nous l'avons dit plus haut, l'abolition graduelle de la distanciation représentative.

\section{BIBLIOGRAPHIE}

Bachelard Gaston, La poétique de l'espace, Paris, PUF, 1957.

Bolter Jay David, Grusin Richard, Remediation, Cambridge, MIT Press, 2000.

Foucault Michel, Dits et écrits II, 1976-1988,1984, Paris, Gallimard.

Latour Bruno, Changer de société, refaire de la sociologie, Paris, La Découverte, 2007.

Lefebvre Henri, La production de l'espace, Paris, Anthropos, 2000.

Lussault Michel, L'Homme spatial. La construction sociale de l'espace humain, Paris, Seuil, 2007.

Scannell Paddy, Radio, Television and Modern Life: A Phenomenological Approach, Wiley, 1996.

Sloterdijk Peter, Bulles. Sphère I, trad. Olivier Mannoni, Paris, Pauvert, 2002.

Sontag Susan, Dans la douleur des autres, Paris, Christian Bourgois éditeur, 2003.

\section{NOTES}

1. Susan Sontag, Dans la douleur des autres, Paris, Christian Bourgois éditeur, 2003.

2. Rainer Maria Rilke, cité par Gaston Bachelard, La poétique de l'espace, Paris, PUF, 1957, p. 183.

3. Michel Foucault, Dits et écrits II, 1976-1988,1984, Paris, Gallimard, p. 1572.

4. Paddy Scannell, Radio, Television and Modern Life: A Phenomenological Approach, Wiley, 1996, p. 76.

5. Henri Lefebvre, La production de l'espace, Paris, Anthropos, 2000.

6. Michel Lussault, L'Homme spatial. La construction sociale de l'espace humain, Paris, Seuil, 2007,

p. 22.

7. Bruno Latour, Changer de société, refaire de la sociologie, Paris, La Découverte, 2007, p. 58.

8. Jay David Bolter, Richard Grusin, Remediation, Cambridge, MIT Press, 2000.

9. Peter Sloterdijk, Bulles. Sphère I, trad. Olivier Mannoni, Paris, Pauvert, 2002, p. 80.

10. Voir : http://www.albertinemeunier.net/060815/index.htm. 


\section{RÉSUMÉS}

La technologie numérique favorise à bien des égards l'émergence d'une nouvelle figuration du désastre. Qu'il s'agisse d'une remédiation vidéo-ludique de l'expérience catastrophiste (via l'interface du jeu vidéo), d'une virtualisation du paysage cataclysmique (via l'imagerie de synthèse - ou le CGI - au cinéma) ou de la mise en circulation de scènes de dévastation (via la mobilité du dispositif portable), nombreux sont les appareils médiatiques actuels qui contribuent de la sorte à composer une nouvelle iconosphère du désastre susceptible, entre autres, de redéfinir les lieux d'émergence de l'espace traumatique. Aussi, si la technologie analogique et les nombreux clichés insoutenables qu'elle nous a légués nous ont appris à nous désoler devant la douleur des autres (S. Sontag), la technologie numérique nous apprend pour sa part à vibrer dans la douleur des autres.

Afin d'illustrer l'actualité et les implications esthétiques de cette spatialisation inédite du trauma et, par voie de conséquence, de mieux comprendre la culture numérique dont elle est le symptôme, nous prendrons ici pour exemple le régime d'imagéité spécifique au "téléphone intelligent " de $3^{\mathrm{e}}$ ou de $4^{\mathrm{e}}$ génération. Nous tenterons, d'une part, de saisir en quoi l'appareil numérique portable instaure une nouvelle «pratique spatiale » de la dévastation, et, d'autre part, d'analyser comment cette pratique parvient à instituer au sein de la culture visuelle contemporaine une véritable mobilité du désastre. Une mobilité qui a pour conséquence première d'introduire au sein de l'iconosphère les concepts d'acteur et de réseau, et qui, afin d'être appréhendée dans toute sa complexité, commande l'élaboration d'une théorie visuelle de l' écumisation médiatique (P. Sloterdijk).

\section{INDEX}

Mots-clés : désastre, espace, images, téléphone intelligent, violence

\section{AUTEUR}

\section{RICHARD BÉGIN}

Professeur adjoint, département d'histoire de l'art et d'études cinématographiques, Faculté des arts et des sciences, université de Montréal (Québec). 